\title{
Nanopropulsion by Biocatalytic Self-Assembly
}

Joy Leckie, ${ }^{1}$ Alexander Hope, ${ }^{1}$ Meghan Hughes, ${ }^{2,3}$ Sisir Debnath, ${ }^{2}$ Scott Fleming, ${ }^{2}$ Alastair W. Wark, $^{2}$ Rein V. Ulijn, ${ }^{2,3+}$ and Mark D. Haw ${ }^{1 *}$

1 - Department of Chemical and Process Engineering, 75 Montrose Street, University of Strathclyde, Glasgow G11XJ, United Kingdom

2 - WestCHEM/Department of Pure and Applied Chemistry, Thomas Graham Building, 295 Cathedral Street, University of Strathclyde, Glasgow G1 1XL, United Kingdom

3 - Advanced Science Research Center (ASRC) and Hunter College, City University of New York, New York, NY1003, USA

* e-mail: mark.haw@strath.ac.uk

† e-mail: rein.ulijn@asrc.cuny.edu

KEYWORDS nanopropulsion; biocatalysis; self-assembly; aromatic peptide amphiphiles, single particle tracking

ABSTRACT A number of organisms and organelles are capable of self-propulsion at the microand nano-scales. Production of simple man-made mimics of biological transportation systems may prove relevant to achieving movement in artificial cells and nano/micronscale robotics that 
may be of biological and nanotechnological importance. We demonstrate the propulsion of particles based on catalytically controlled molecular self-assembly and fiber formation at the particle surface. Specifically, phosphatase enzymes (acting as the engine) are conjugated to a quantum dot (the vehicle), and are subsequently exposed to micellar aggregates (fuel), that upon biocatalytic dephosphorylation undergo fibrillar self-assembly, which in turn causes propulsion. The motion of individual enzyme/quantum dot conjugates is followed directly using fluorescence microscopy. While overall movement remains random, the enzyme-conjugates exhibit significantly faster transport in the presence of the fiber forming system, compared to controls without fuel, a non-self-assembling substrate or a substrate which assembles into spherical, rather than fibrous structures upon enzymatic dephosphorylation. When increasing the concentration of the fiber-forming fuel, the speed of the conjugates increases compared to nonself-assembling substrate, although directionality remains random.

Self-propulsion of micro- and nano-scale objects is an area of research that has become increasingly popular in recent years as model systems of biological transport systems, to enable motility in cell mimics and more generally to study how chemical energy can be converted into mechanical energy. ${ }^{1-14}$ The inspiration for artificial 'nanomotors' comes from motile biological organisms and organelles. Biological systems use motor proteins and their associated filaments or 'tracks' for motility and transportation of cargo within the cell. Whole cells are also capable of directed movement using catalytic self-assembly mechanisms such as actin polymerization. Actin polymerization and motor protein action are key examples of how biology converts chemical energy, in the form of a molecular fuel such as adenosine triphosphate (ATP), into mechanical energy to achieve motion. 
The majority of examples of artificial self-propelled nanoscale and microscale objects employ non-biological components. The first catalytic motor was reported by Whitesides et al. using millimeter sized plates propelled at the air/water interface, by the decomposition of hydrogen peroxide on the platinum surface of the motor. ${ }^{15}$ The mechanism of this system subsequently inspired the development of small scale (micro- and nanometer sized) motors based on the same principle. Indeed, smaller metallic catalytic motors have been studied widely for propulsion using metal catalysts, such as platinum in hydrogen peroxide solutions, by oxygen bubble, ${ }^{2-4}$ electrochemical $^{5,6}$ or surface tension-induced ${ }^{7,8}$ mechanisms. A recent example uses a combination of catalysis and polymerization to drive motion, giving rise to polymerizationpowered motors, achieved using ring opening metathesis polymerization (ROMP) at the particle surface. ${ }^{1}$

A number of synthetic/biological hybrid approaches incorporate biological catalysts to perform similar reactions to metal catalysts. Enzymes have a number of potential advantages in this regard due to selectivity, compatibility with aqueous conditions and their remarkable turnover rates. Based on the hydrogen peroxide propulsion discussed above, the enzymes glucose oxidase and catalase have been employed for the biocatalytic propulsion of artificial objects. ${ }^{9-11}$ Additionally a number of 'biohybrid' examples of artificial objects propelled by actin polymerization have been described. ${ }^{16-18}$ Researchers have also taken advantage of biological components known for their inbuilt motor activity i.e. kinesin-microtubule and myosin-actin motor protein-filament pairs. . $^{12,13,19,20}$

Our approach originated in the observation that biocatalytic self-assembly provides a potential route to the production of a propulsion mechanism. We previously demonstrated that during the catalytic formation of self-assembly building blocks, in this cased produced by enzymatic 
condensation of non-assembling precursors to produce a self-assembling aromatic peptide amphiphile, catalysis and self-assembly is co-localised. This is especially the case if the selfassembling molecule produced has a poor solubility, giving rise to a kinetic preference to assemble locally, at the site of formation. ${ }^{21}$ A similar localized catalytic assembly effect was recently exploited for localized catalytic gelation by Van Esch and colleagues, ${ }^{22}$ and also underpinned the formation of localized nanoscale networks produced by biocatalytic assembly by phosphatase enzymes at the cell surface by $\mathrm{Xu}$ et $a l .{ }^{23}$ The above observations were made by using catalysts immobilized onto synthetic or biological surfaces. We decided to investigate whether propulsion could be achieved by adapting this approach to particles to achieve propulsion which is inspired by actin polymerization, but is much simpler and potentially more versatile considering the wide variety of enzymatic systems that could potentially be exploited.

Thus, we develop a biohybrid motor which incorporates the efficiency and selectivity of biological catalysis, to trigger a self-assembly-based propulsion mechanism. Instead of the polymerization of actin monomers into filaments, smaller aromatic dipeptide amphiphiles are investigated for biocatalyzed supramolecular self-assembly into fibrous structures and consequent propulsion. Previously, self-assembly of dipeptides was reported by Matsui et al. for the autonomous propulsion of metal organic frameworks (MOF). ${ }^{24}$ In this case propulsion was powered by the reorganization of self-assembling peptides (diphenylalanine, $\mathrm{FF}^{25}$ ) at the MOF interface (whereby the MOF acts as a reservoir and release device for the dipeptide fuel) in comparison with our biocatalytic approach.

Aromatic peptide amphiphiles are short peptides that are capped with an aromatic N-terminal group e.g. Fmoc, napthalene or pyrene. ${ }^{26-31}$ Self-assembly of these molecules is a result of 
aromatic stacking interactions between the aromatic groups and hydrogen bonding between peptide chains. ${ }^{32-34}$

Biocatalytic self-assembly has been reported over recent years with a variety of enzymes and peptidic substrates. ${ }^{35,36}$ Aromatic amphiphiles offer a versatile platform for biocatalytic selfassembly due to their chemical simplicity they can be easily synthesized and functionalized with groups recognized as substrates. In particular, incorporation of phosphorylated tyrosine groups which can be cleaved by alkaline phosphatase, dramatically changes the hydro-phobic/philic balance, enabling conversion of non-assembling precursors to self-assembly building blocks. Phosphatase triggered self-assembly was first reported by $\mathrm{Xu}$ et $a l^{37,38}$ for fluorenyl functionalized phosphorylated tyrosine residues (Fmoc- $p \mathrm{Y}$ ). Since this early report, biocatalytically triggered self-assembly of aromatic peptide amphiphiles has developed into an active field, with examples studied in the context of biomedical, electronic and drug delivery applications, but so far it has not been associated with motility applications..$^{30,38-44}$

Here we investigate the propulsion of the enzyme alkaline phosphatase (EC. 3.1.3.1) from Escherichia coli, using the biocatalytically induced self-assembly of an aromatic peptide amphiphile, fluorenylmethoxycarbonyl-phenylalanine-phosphotyrosine(Fmoc-Fp $\mathrm{Y})^{39,42,45}$ (1a) see Figure 1. The amphiphilic nature of 1a results in formation of spherical micelle structures in water. ${ }^{45}$ Enzymatic removal of the charged tyrosine phosphate group, leads to the dephosphorylated product 1b (Figure 1.), which self-assembles into $\beta$-sheet-like fibrous nanostructures, as described previously. ${ }^{45}$

In order to determine and analyze the motion of the enzyme conjugates a method was required to track the conjugates over time by visualizing individual vehicles. In principle this can be 
achieved by labeling the enzyme to enable observation via fluorescence microscopy. It is a significant challenge to visualize and track single nanosized objects in a three dimensional, potentially optically turbid environment, compared for example to studies of particle tracking confined to surfaces or interfaces i.e. a 2D environment. Methods for visualization of single proteins include labeling them with organic fluorophores, ${ }^{46}$ genetic fusion with a naturally fluorescing protein ${ }^{47}$ or conjugation to nano- or micro-sized fluorescent particles. ${ }^{48-50}$ Organic fluorophores generally result in insufficient fluorescence emission for adequate time resolution for single molecule tracking, ${ }^{51}$ and may suffer from considerable photobleaching after only a few minutes illumination. ${ }^{52}$ Conjugation to micron-sized particles, though more easily trackable by optical microscopy, would involve a significant load on the biomolecule potentially resulting in sedimentation rather than Brownian diffusion. Nanoparticles (NPs), on the other hand, are comparable in size to many proteins. Metal NPs, i.e. gold or silver are useful for tracking using optical phase contrast or differential interference contrast (DIC) microscopy,${ }^{53}$ however they do not emit light so are unsuitable for fluorescence microscopy. ${ }^{48,51}$ Nanosized semiconductor quantum dots $(\mathrm{QDs})^{54}$ have a number of advantages for microscopy imaging applications, compared to organic fluorophores and fluorescent proteins, resulting in their potential for use in suspension: QDs are 10-100 times brighter, due to their large molar extinction coefficients between $0.5-5 \times 10^{6} \mathrm{M}^{-1} \mathrm{~cm}^{-1} ; 52,55,56$ and they are generally highly photostable, enabling long-term tracking. ${ }^{55,57}$ Therefore in this study we make use of enzyme-QD conjugates for tracking and visualization. The specific objectives of this work are (i) to produce bioactive QD-enzyme conjugates, (ii) track their motion in response to three different phosphorylated fuels a fiber assembling unidirectional substrate (1a), nondirectional sphere assembling (3a) and non- 
assembling $p$-nitrophenyl phosphate (2a) and (iii) determine the effect of fuel concentration on motility (1a).

\section{Results and Discussion}

\section{Enzyme-QD conjugation}

The first objective was to conjugate the enzyme to the QD for visualization in such a way that neither enzyme activity nor QD fluorescence are compromised. Alkaline phosphatase from Escherichia coli was conjugated to $655 \mathrm{~nm}$ carboxyl- functionalized quantum dots via EDC (Ethyl-3-[3-dimethylaminopropyl]carbodiimidehydrochloride)/ NHS (N-Hydroxysuccinimide) coupling (Figure S1) then purified by multiple wash steps, followed by filtration and centrifugation to remove free enzyme and coupling reagents. To ensure all free/unreacted enzyme had been removed from the conjugate the purification wash samples were analyzed using a colorimetric assay (Figure S2) consisting of dephosphorylation of the substrate $p$ nitrophenyl phosphate, resulting in a yellow color change which can be monitored at $410 \mathrm{~nm}$ by UV-VIS spectroscopy. After 8-12 washes the absorbance of the wash at $410 \mathrm{~nm}$ was reduced to the value obtained for the negative control, confirming that all free phosphatase had been removed.

The enzyme-QD conjugates were subsequently characterized by dynamic light scattering (DLS) (Figure S1). Before conjugation, the average hydrodynamic radius of alkaline phosphatase and QDs were found to be approximately $8 \mathrm{~nm}$ and $10 \mathrm{~nm}$ respectively, (in agreement with the dimensions for the crystal structure of alkaline phosphatase $10 \times 5 \times 5 \mathrm{~nm}^{58}$ and $12 \times 6 \mathrm{~nm}$ for free QDs, as quoted by manufacturer). DLS of the conjugate (QD-AP) solution revealed a 
hydrodynamic radius centered around $17 \mathrm{~nm}$ along with a larger size distribution ranging between 2-150 nm hydrodynamic radius (Figure S1).

The fluorescence emission spectra of the free QDs and the QD-AP conjugate were compared to show that the maximum emission of the QDs $\left(\lambda_{\max }=650 \mathrm{~nm}\right)$ was unaffected by the conjugation (Figure $\mathrm{S} 1$ ). We estimate that there is approximately a 2.8:1 ratio of QD to enzyme in the QDAP conjugate solution, assuming that the base activity of the enzyme and the fluorescence emission intensity of the QDs are unaffected by the conjugation reaction (see figures S5, S9 and S11 for method used to estimate QD-AP ratio and batch variation). Long term stability of the conjugates was measured via the rate of p-nitrophenyl phosphate dephosphorylation (i.e. the change in absorbance at $410 \mathrm{~nm}$ over time), showing steady activity over at least eight weeks, when stored below $4{ }^{\circ} \mathrm{C}$ (Figure S6).

\section{Enzyme-conjugate tracking analysis}

Fluorescence microscopy was used to visualize solutions containing QD-AP conjugates. In order to visualize and track single conjugates, an EMCCD camera fitted with a $650 / 60 \mathrm{~nm}$ optical filter was employed. The excitation wavelength chosen for this study was $435 \mathrm{~nm}$ (lower wavelengths e.g. $365 \mathrm{~nm}$ can also be used, however a compromise must be made between more efficient excitation of QDs at lower wavelengths and background fluorescence/scattering issues due to the fluorescent nature of the self-assembling fuels in the UV range). Two batches of QD-AP conjugates were used to accumulate all of the data presented (the effect of batch variation is discussed further below and in figures S5, S9 and S11). We note that individual experiments are time consuming (typically 3-5 days to obtain experimental data, convert files, analyze via MATLAB and plot results, per repeat) and cannot be carried out in parallel, therefore we ensure 
statistical validity of the results by collecting large amounts of data from many thousands of individual particle tracks. Control data was obtained using both batches.

QD-AP conjugates were added to a solution of the relevant substrate/fuel (1a, 2a or 3a) in a glass bottom dish (Figure 2). (Note that the conjugate and substrate concentration is low such that fiber growth does not lead to gelation on the timescale of the motility measurements.) Tracking videos obtained of enzyme-conjugates are composed of a sequence of frames. The position differences between specific particles in neighboring frames are analyzed by a modified MATLAB program to obtain data including particle trajectory (Figure 2.), frame-to-frame speed, mean square displacement (MSD) and angle of trajectory, for the three substrates and the control without fuel, which measures free diffusion of the QD-AP conjugate for comparison. Each video analyzed was a total of 300 or 600 frames in duration at a rate of typically 23 frames per second. Since particles move in and out of the plane of focus, individual tracks can be of varying length during the video. To check whether activity changes over time, videos were captured at specific time points over a period of 60 minutes i.e. every 2-5 minutes. The number of particles tracked in each video varied from 5 to 200, and each experiment was repeated up to 4 times. In cases where the average speed of particles per video stayed constant over time, the frame-to-frame speed data for all frames was combined and an overall distribution of speeds and average overall value was obtained. The number of particles tracked is thus a combination of repeated experiments, and the nature of the experiment allows for specific particles to be counted multiple times as new particles if they leave the focal plane then return at a later time point. Video microscopy carried out in this way provides a wealth of data: while further detailed statistical analysis will be presented elsewhere, in this paper we focus on the distribution of frame-to-frame speeds, to 
determine whether motion is enhanced in the biocatalytic self-assembling system, i.e. whether biocatalytic self-assembly can be employed to achieve nanoscale propulsion.

Example trajectories of tracked conjugates for the self-assembling 1a and non-self-assembling 2a substrates are shown in Figure 2. Trajectory examples for the nondirectional self-assembling substrate (3a) and for the control (no substrate) are shown in Figure S7. Clearly motion remains diffusive on the length-scale (microns) and time-scale (seconds) observed here. This is entirely expected: micellar substrate fuel molecules will be encountered at random by the QD-AP conjugates and bursts of motion driven by fibre assembly will be in random directions. However, in the presence of a $10 \mathrm{mM}$ self-assembling fuel 1a solution, QD-AP conjugates exhibit enhanced rate of diffusion: with an average frame-to-frame speed of $5.7 \mu \mathrm{m} / \mathrm{s}$ (based on 7007 particle trajectories and 4 experiments), and top speeds up to $27.1 \mu \mathrm{m} / \mathrm{s}$, compared to the no-fuel control of $4.4 \mu \mathrm{m} / \mathrm{s}$ (719 particle trajectories and 3 experiments) with top speed of $19.6 \mu \mathrm{m} / \mathrm{s}$ i.e. an enhancement of average frame-to-frame speed of $29 \%$. Additionally, the non-assembling fuel 2a has no significant effect on the speed of the conjugates compared to the no-fuel control (average instantaneous frame-to-frame speed of $4.0 \mu \mathrm{m} / \mathrm{s}$ and top speeds of $22.6 \mu \mathrm{m} / \mathrm{s}$ for 1139 particles and 2 experiments). Conjugates with 3a also show no evidence of enhanced motion, (average frame-to-frame speed of $4.6 \mu \mathrm{m} / \mathrm{s}$ and top speed of $20.7 \mu \mathrm{m} / \mathrm{s}$ based on 1131 particle trajectories and 2 experiments), despite a faster initial conversion rate for $\mathbf{3 a}$, compared to $\mathbf{1 a}$ as shown in Figure S10. This implies that indeed fiber formation i.e. asymmetric unidirectional selfassembly, by 1a converting to $\mathbf{1 b}$ is responsible for the enhanced diffusion (Figure 3.). Moreover, Figure 4. shows that for fuel (1a) the frame-to-frame speed distribution shifts to faster speeds with fuel concentration (at least up to $10 \mathrm{mM}$ fuel concentration, see below for further discussion of behavior at higher fuel concentration) while with non-self-assembling fuel (2a) 
measured average speed is independent of fuel concentration. Diffusion coefficient (D) values for conjugates were determined using the average mean square displacement (MSD) of conjugate populations using the following equation:

$$
M S D_{x y}=4 D
$$

The average diffusion coefficient values for QD-AP conjugates without fuel and in the presence of $10 \mathrm{mM} \mathrm{1a}, 2 \mathrm{a}$ and 3a were found to be $1.42 ; 2.71 ; 1.49$ and $1.99 \mu \mathrm{m}^{2} / \mathrm{s}$ respectively, indicating faster diffusion with fiber assembling fuel 1a. Similar trends have been observed by Sen et al. using fluorescence correlation spectroscopy to measure diffusion coefficients of enzyme molecules with and without substrate. ${ }^{59}$

While average speeds and average diffusion coefficients are informative, the DLS analysis of the conjugate clearly revealed a range of conjugate sizes, so within a population a range of rates of motion can be expected. In Figure 3 we compare frame-to-frame speed distributions for the selfassembling and non-self-assembling substrates (at $10 \mathrm{mM}$ fuel concentration) and the no-fuel control. The self-assembling fuel 1a shows a clear and significant population of faster particles compared to the no fuel control, the self-assembling control (3a) and non-self-assembling fuel (2a); moreover the peak in the speed distribution is significantly shifted to higher values (Figure 3), further supporting the case that biocatalytic self-assembly into fibrous structures drives enhanced motion. QD-AP conjugates from the same batch are used in all experiments; hence the distribution of sizes of conjugates and how many enzymes are attached per QD will be statistically similar for all different substrates: if motion were not enhanced by the presence of the fiber forming fuel substrate, we would expect to see overlapping distributions for all cases in Figure 3. 
While we see a clear increase in enhancement of motion with increasing fiber self-assembling fuel (1a) concentration between $5 \mathrm{mM}$ and $10 \mathrm{mM}$ and frame-to-frame speed distributions exhibit populations of conjugates moving at faster rates compared to non-self-assembling fuel (2a) (Figure 4). Interestingly at $20 \mathrm{mM}$ 1a the frame-to-frame speed distribution reverts to slower conjugates, however speeds remain enhanced compared to the control and non-self-assembling fuel, they are reduced compared to $10 \mathrm{mM}$ fiber-assembling fuel 1a (Figure 4). Given the lack of information about the detailed mechanism, the dependence on fuel concentration is difficult to explain definitively; however it is worth noting that at higher concentrations of 1a e.g. $16 \mathrm{mM}$, Fmoc-FpY self-assembly has been observed to occur without catalysis. ${ }^{60}$ This would imply that at higher concentration some proportion of the 'fuel' was unavailable since it had already converted to a self-assembled nano-rod state. In addition, increased viscosity may play a role here which counteracts the increase in fuel concentration.

In Figure S8, frame-to-frame speed distributions of two enzyme-conjugate batches are compared (with $10 \mathrm{mM} \mathrm{1a),} \mathrm{to} \mathrm{illustrate} \mathrm{that} \mathrm{batch-to-batch} \mathrm{variations} \mathrm{exist.} \mathrm{Faster} \mathrm{conjugates} \mathrm{are} \mathrm{observed}$ for the more active (in terms of $p$-nitrophenyl phosphate turnover) QD-AP (batch 3) (Figure S5). Differences in activity are likely due to differences in the degree of conjugation of enzyme to QDs (batch variation shows QD:AP ratios of 2.8:1 vs. 13:1 (see S11)). Additionally experimental repeatability is confirmed for QD-AP conjugates (batch 6), with $10 \mathrm{mM} \mathrm{1a}$, performed on different days (Fig. S8).

Significant questions remain as to the details of the mechanism of propulsion. Efforts to simultaneously visualize a conjugate and the self-assembly into fibers were not successful. While Fmoc-based fiber systems do not fluoresce at an accessible wavelength, extrinsic staining methods using thioflavin $\mathrm{T}$ and congo red have proven insufficient to visualize dilute samples 
containing localized regions of short fiber growth. In future, it may be possible to use high resolution methods, such as Stochastic Optical Reconstruction Microscopy (STORM) confocal microscopy to image fibril formation in real time ${ }^{61}$ However, given the trajectories shown, it appears that individual fiber-growth propulsion 'events' take place on very short timescales, and that the overall enhanced motion, observed here on micron length-scales and time-scales of seconds, is the result of many of these events, each one providing a small extra 'impulse' in the conjugate's random walk. Direct visualization of such short-timescale and nm-length scale events would be a major challenge. Methods to measure forces involved during the self-assembly process and the propelled motion, such as via optical tweezing or force microscopy, perhaps offer one way to further investigate and refine the mechanism at the level of a single driven conjugate. ${ }^{62,63}$

\section{Conclusions}

In summary it has been shown that the diffusive transport of alkaline phosphatase-quantum dot conjugates is enhanced by the addition of a self-assembling fuel 1a. The fiber self-assembling fuel, 1a is dephosphorylated by the enzyme to $\mathbf{1 b}$, which facilitates fiber formation, in turn propelling alkaline phosphatase-QD conjugates and leading to enhanced motion. In the case of the self-assembling control 3a, which assembles into spherical aggregates rather than fibrous structures, enhanced diffusion was not observed. This leads to the conclusion that, with fibers potentially providing a higher surface tension gradient compared to spherical aggregates and thus increased directed propulsive force, asymmetric growth of self-assembled $\mathbf{1 b}$ molecules into linear fibers is essential for the nanopropulsion of QD-AP conjugates. 
Such a system could be used for cargo transportation, nano-separation of enzymes and also potentially allows information to be gained on enzyme motion and forces during catalysis. Control of enhanced motion could be realized using gradients of self-assembling fuel concentration or, in analogy to microtubule cytoskeleton in biology, 'templated' tracks releasing local supplies of fuel. Moreover, by designing different peptide fuel-enzyme motor pairs, where motors have affinity to different molecular or particulate cargo, one could realize systems of highly selective driven nanoscale transport, with all the efficiency and selectivity advantages of biological catalysts. Finally, enzyme-peptide systems are also available with dynamically unstable self- assembly, ${ }^{31}$ potentially enabling 'recharging' of fuel. 


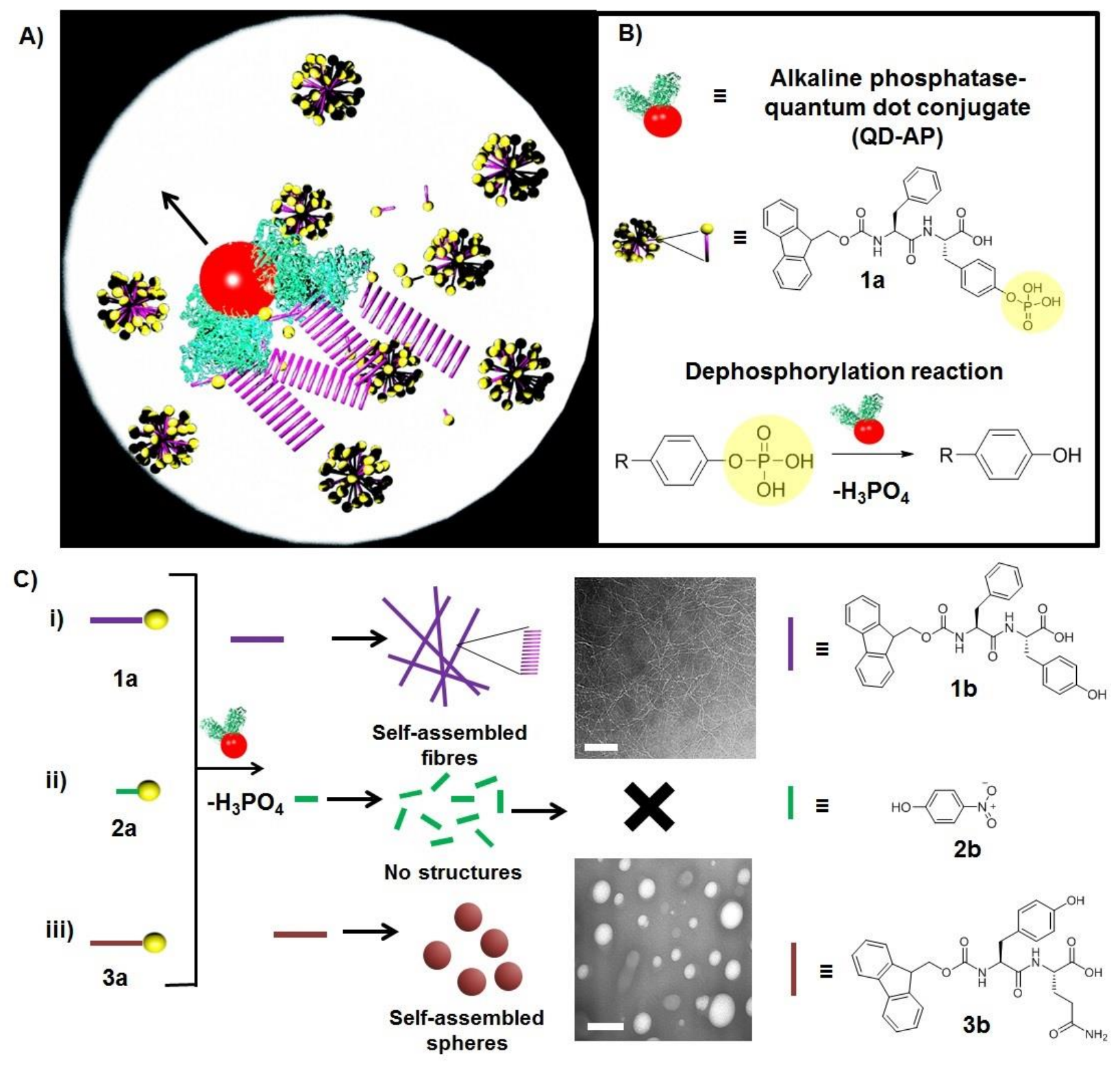

Figure 1. A) Scheme illustrating an alkaline phosphatase-quantum dot conjugate propelled by the biocatalytic transformation of micellar fuel molecules of $\mathbf{1 a}$ into fiber structures of $\mathbf{1 b}$ (scheme not to scale). B) Key illustrating alkaline phosphatase-QD conjugates and micellar fuel molecules of Fmoc-FpY (1a), including a general biocatalytic dephosphorylation reaction for all fuels. C) i) Fiber assembling fuel 1a, ii) non-self-assembling control fuel 2a; and iii) sphere assembling fuel $\mathbf{3 a}$, converted by QD-AP to $\mathbf{1 b}, \mathbf{2} \mathbf{b}$, and $\mathbf{3 b}$ respectively. TEM images of fibers and spheres obtained using free AP (scale bars $=200 \mathrm{~nm}$ ). 
A)

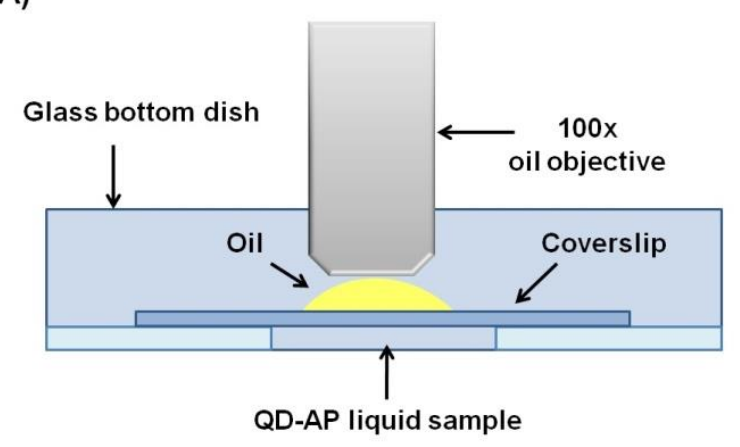

C)

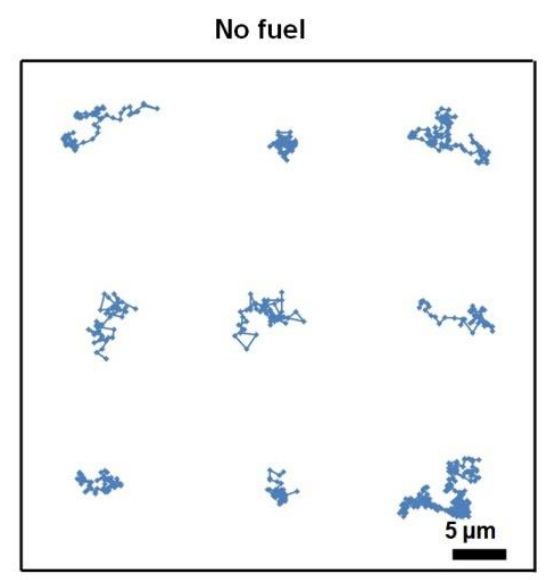

B) Example trajectory
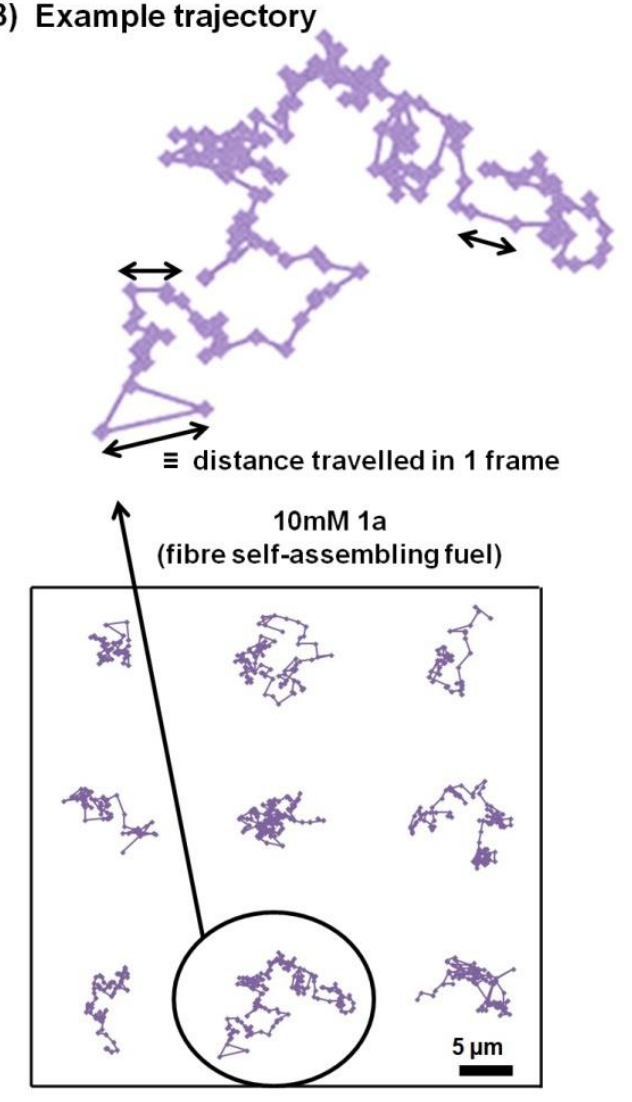

Figure 2. A) Experimental set-up of motility assay. A solution of QD-AP conjugates is added to a glass bottom dish (with/without fuel). A glass coverslip is added to seal the chamber and oil added on top of the coverslip for use with an 100x oil immersion objective. B) Example trajectory of a single QD-AP conjugate 'fuelled' by $10 \mathrm{mM}$ 1a. Each connected pair of data points represents the distance travelled between subsequent frames. This data is then used to calculate the 'frame-to-frame' speed. C) Composites of separate example trajectories for QD-AP with no fuel and in the presence of $10 \mathrm{mM} 1 \mathrm{a}$ (other control trajectories can be found in Figure S7)). 
A)

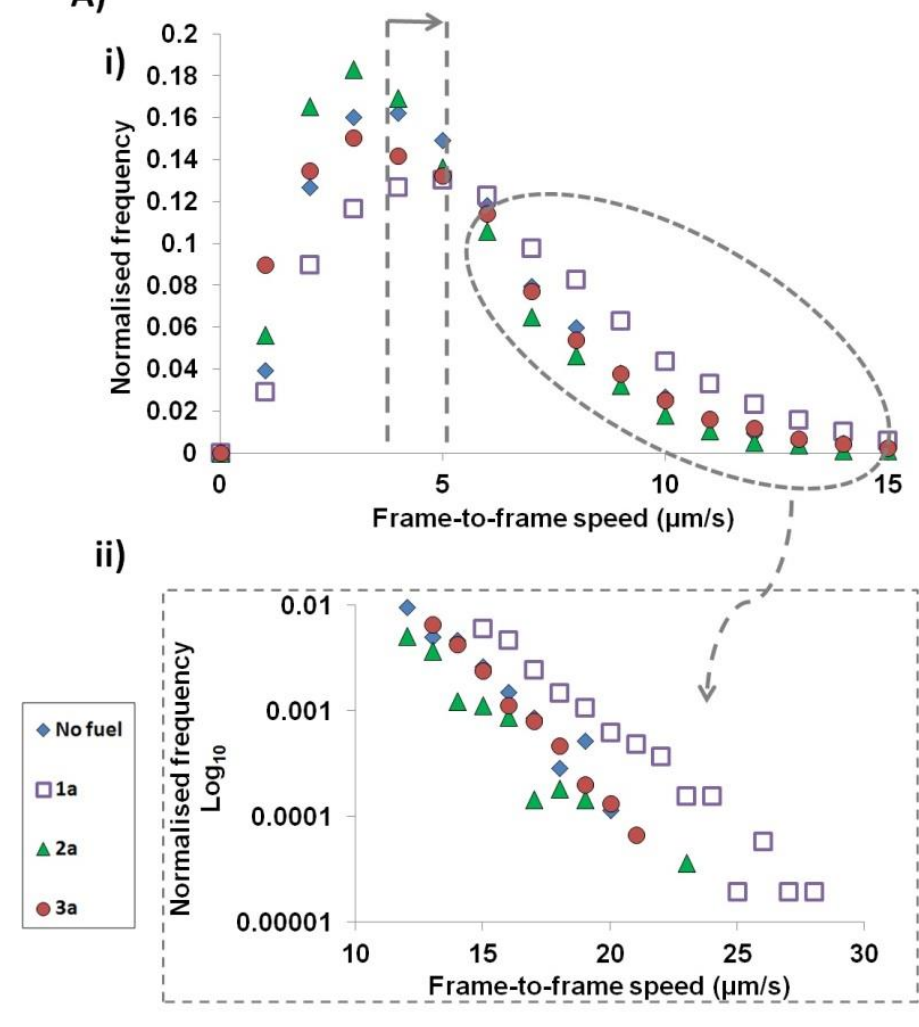

B)

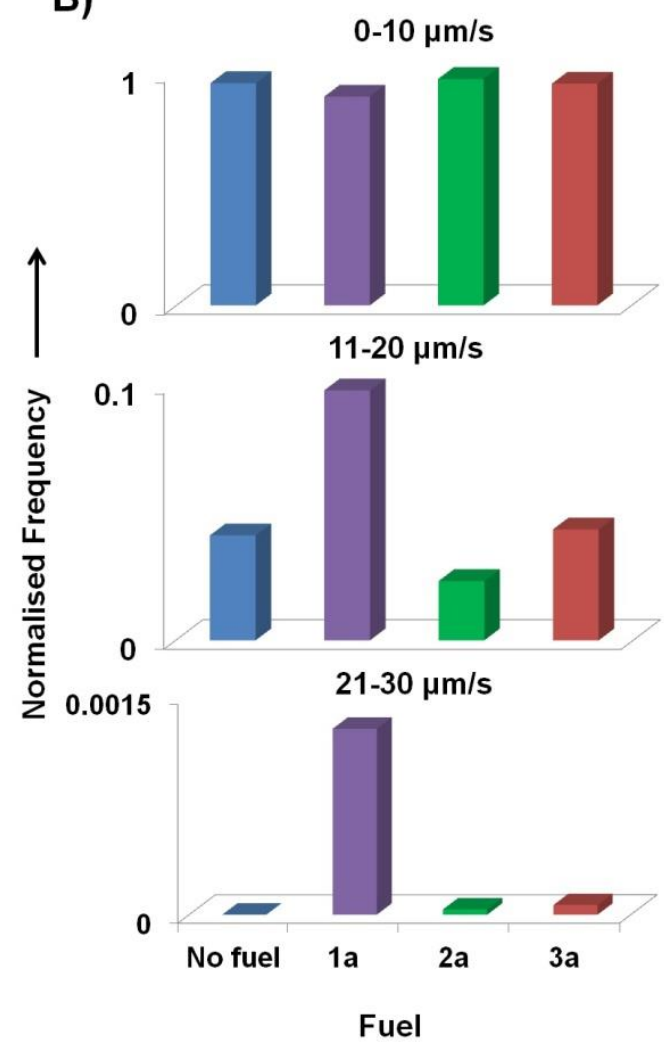

Figure 3 - A) i)Frame-to-frame speed distribution of QD-AP conjugates with different fuels and without fuel: 1a - fiber self-assembling fuel; 2a - non-self-assembling fuel, 3a spherical selfassembling fuel. A significant shift in the population maxima is observed with 1a, compared to no fuel, 2a and 3a, indicative of a faster average frame-to-frame speed. Dotted lines illustrate the peak maxima and shift for QD-AP conjugates with no fuel compared to with 1a. ii) Frame-toframe speed distribution (log scale) illustrating a significant population of conjugates at higher speeds with $10 \mathrm{mM}$ 1a. B) Histograms representing populations of conjugates for each fuel scenario at speed ranges from 0-10;11-20 and 21-30 $\mu \mathrm{m} / \mathrm{s}$. With the fiber self-assembling fuel 1a, there are fewer conjugates moving at the lower speed range $(0-10 \mu \mathrm{m} / \mathrm{s})$, compared to other fuels (and no fuel), while more conjugates, display faster frame-to-frame speeds between 11-20 and $21-30 \mu \mathrm{m} / \mathrm{s}$ speed ranges. 
A)

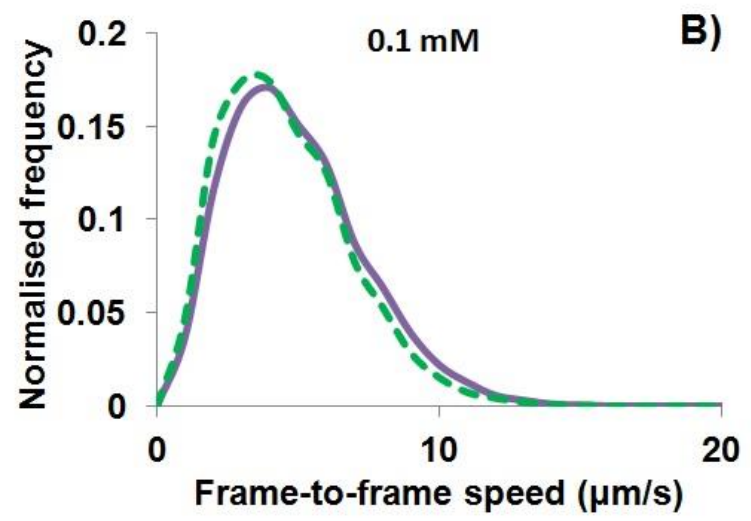

C)

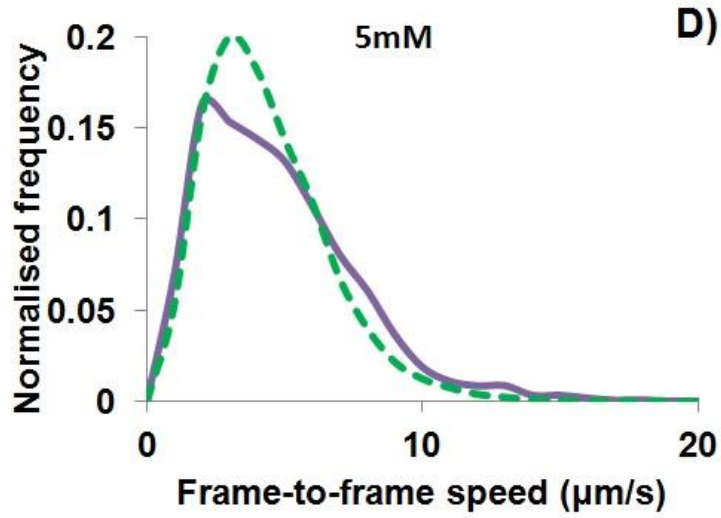

E)

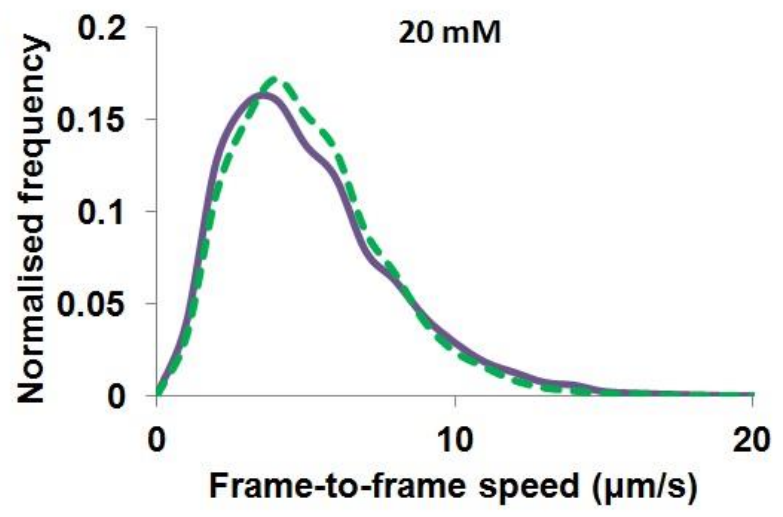

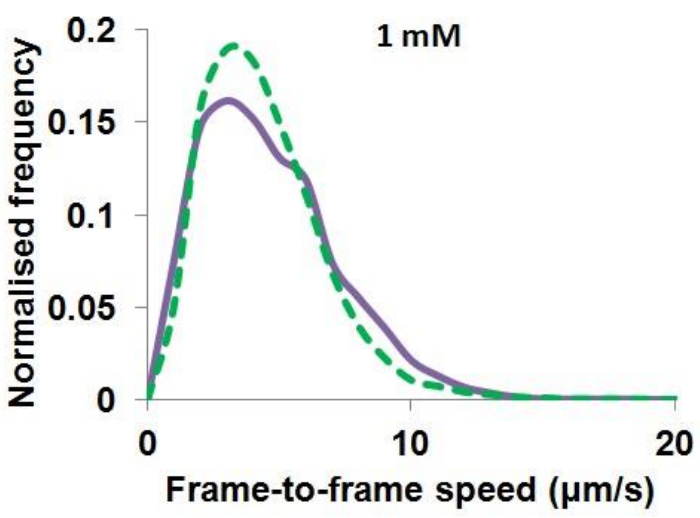

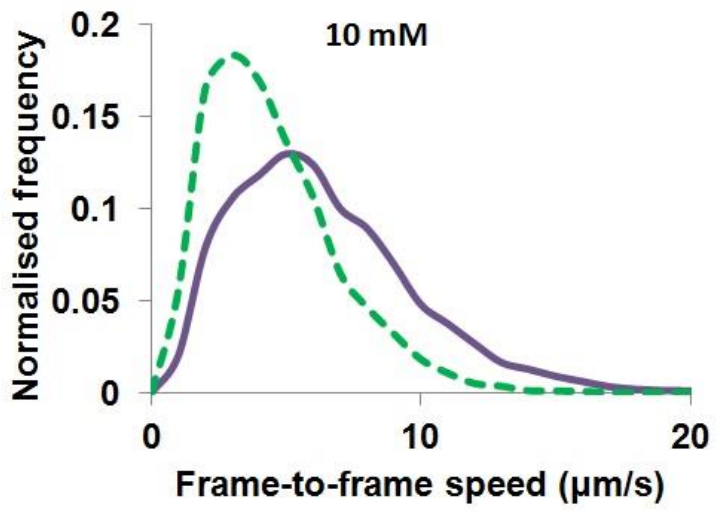

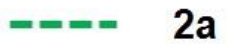

Figure 4 - Frame-to-frame speed distributions of QD-AP conjugates with different concentrations of fiber assembling fuel 1a (purple solid line) and non-self-assembling substrate 2a (green dashed line). 


\title{
ASSOCIATED CONTENT
}

\author{
Funding Sources
}

This work was funded by Engineering and Physical Sciences Research Council (EPSRC).

M.D.H acknowledges support from the Leverhulme Trust. The research leading to these results has received funding from the European Research Council under the European Union's Seventh Framework Programme (FP7/2007-2013) / ERC grant agreement n [258775].

\section{METHODS}

All reagents were purchased from commercial sources at the highest purity and were used as supplied, unless stated otherwise in the experimental procedures. Fmoc-FpY was purchased from CS Bio Co., Fmoc-pYQ was synthesized by S.D, ${ }^{39}$ alkaline phosphatase (Escherichia coli) was purchased from Sigma Aldrich and quantum dots from Invitrogen (Q21321MP Lot: 891174 and 1252823).

Alkaline phosphatase-quantum dot conjugation and purification

The enzyme-QD conjugation method was adapted from the Invitrogen bioconjugation method. ${ }^{64}$ A $0.85 \mu \mathrm{M}$ QD solution was prepared in $10 \mathrm{mM} \mathrm{pH} 7.4$ borate buffer. A reaction mixture was prepared by a combing EDC activated quantum dots, with alkaline phosphatase (Sigma Aldrich P5931 lyophilized powder, 30-60 units/mg protein (in glycine buffer))and NHS at a molar ratio of 1:13:1500:6500 (QDs:AP:EDC:NHS). The reaction mixture was left to react on a blood rotator for 2 hours at room temperature. The conjugate was filtered through a $0.2 \mu \mathrm{m}$ PES syringe filter into an Amicon Ultra centrifugal ultrafiltration unit with a $100 \mathrm{kDa}$ cutoff 
(Millipore), and then diluted with $2.5 \mathrm{~mL}$ of borate buffer. The conjugates were centrifuged in an Eppendorf centrifuge 5415R at 10,000 rpm for 5 minutes and then washed with borate buffer up to 12 times (until no catalytic activity was measured in the wash), whilst retaining each of the wash samples for further analysis. The purity of the conjugate was determined by adding $50 \mu \mathrm{L}$ of each wash sample into a $1 \mathrm{mM}$ concentration of $p$-nitrophenyl phosphate in $1.5 \mathrm{M} \mathrm{pH} 8$ Tris buffer. The dephosphorylation reaction was monitored by UV-VIS (Jasco V660 spectrophotometer) by recording the absorbance at $410 \mathrm{~nm}$ over 10 minutes (method also used for activity assay of pure conjugate). After the wash sample absorbance resembled that of the negative control the conjugate was deemed pure (see supplementary figure 2).

The pure conjugate was then transferred and filtered through a $0.2 \mu \mathrm{m}$ syringe filter and diluted by the addition of $4 \mathrm{~mL}$ of $50 \mathrm{mM} \mathrm{pH} 8.3$ borate buffer. The final conjugate solution was stored at $4^{\circ} \mathrm{C}$.

Dynamic Light Scattering (DLS)

DLS was performed on an AVL/LSE-5004 light scattering electronics and multiple tau digital correlator. Solutions of QDs, free AP and QD-AP conjugates were filtered, using a $0.2 \mu \mathrm{m}$ syringe filter, into glass tubes and analyzed using a DLS instrument at a temperature of $295 \mathrm{~K}$, with an angle of $90^{\circ}$ and wavelength of $632 \mathrm{~nm}$.

Atomic force microscopy (AFM)

$20 \mathrm{ml}$ of a QD-AP solution was placed on a trimmed, freshly cleaved mica sheet attached to an atomic force microscopy (AFM) support stub, which was left to air-dry overnight in a dust-free environment, prior to imaging. The images were obtained by scanning the mica surface in air under ambient conditions using a VeecoMultiMode with NanoScope IIID Controller Scanning 
Probe Microscope (Digital Instruments, Santa Barbara, CA, USA; Veeco software Version 6.14r1) operated in tapping mode. The AFM measurements were obtained using a sharp silicon probe $($ TESP; nominal length $($ lnom $)=125 \mathrm{~mm}$, width $($ wnom $)=40 \mathrm{~mm}$, tip radius $($ Rnom $)=8$ $\mathrm{nm}$, resonant frequency $(\mathrm{nom})=320 \mathrm{kHz}$, spring constant $(\mathrm{knom})=42 \mathrm{~N} \mathrm{~m}-1$; Veeco Instruments SAS, Dourdan, France), and AFM scans were taken at $512 \times 512$ pixels resolution. Typical scanning parameters were as follows: tapping frequency $308 \mathrm{kHz}$, integral and proportional gains 0.3 and 0.5 , respectively, set point $0.5-0.8 \mathrm{~V}$ and scanning speed $1.0 \mathrm{~Hz}$. The images were analyzed using Veeco Image Analysis software Version 6.14r1.

Fluorescence spectroscopy

Fluorescence emission spectra were measured on a Jasco FP-6500 spectrofluorometer with light measured orthogonally to the excitation light, with excitation at $295 \mathrm{~nm}$ and emission data range between 300 and $600 \mathrm{~nm}$ for Fmoc-dipeptide samples. For QD/QD-AP samples excitation was $435 \mathrm{~nm}$, bandwidth $3 \mathrm{~nm}$ and emission range between $450-700 \mathrm{~nm}$.

Motility assays

Optical tracking motility experiments were performed using a $35 \mathrm{~mm}$ glass bottom dish (Ibidi®). $60 \mu \mathrm{L}$ of the motility buffer $(0.6 \mathrm{M} \mathrm{pH} 9$ phosphate buffer) containing QD-AP conjugates in 5 $\mathrm{mM}$ fuel (Fmoc-F $p$ Y 1a, $p$-nitrophenol phosphate 2a, Fmoc- $p$ YQ 3a or no fuel) was added to the well in the glass bottom dish. A glass coverslip $(22 \times 22 \mathrm{~mm})$ was placed over the well and immersion oil was added to the coverslip for use with 100x oil immersion objective.

Optical imaging

Video acquisition was performed using a fluorescence microscope (Nikon Eclipse LV100) with a 100x oil immersion 0.5-1.3 NA objective (Nikon Plan Fluor), combined with an AndoriXon+ 
897 EMCCD camera fitted with a $650 / 60 \mathrm{~nm}$ optical filter (Semrock) to capture the signals from the quantum dots. Excitation of quantum dots was performed by a halogen lamp combined with a $435 \mathrm{~nm}$ optical filter (Semrock). Videos were obtained in kinetic acquisition mode with typically 23 frames $\mathrm{s}^{-1}$. We define 'frame-to-frame speed as the speed measured over a 10 frame interval i.e. frames 1-10;2-11;3-12 etc.

Video analysis and processing

Single particle tracking was performed using an in-house programme on MATLAB (Mathworks, Natick, MA). Particle identification and tracking software was used to gather information on trajectories of QDs identified in the plane of focus in a given recorded video. This identification software was based on original IDL code by John Crocker and David Grier. ${ }^{65}$ In each frame of a recorded video, QDs are identified based on the intensity of light emitted compared to the rest of the sample being viewed. Based on the position, apparent shape, size and intensity of a given identified QD, it is assigned an identification number. This allows data to be gathered on multiple QDs simultaneously. The tracking software then calculates the frame-to-frame displacement of each identified QD within the field of view, over the entire video or until it moves out of the plane of focus.

\section{Cryo TEM}

Three microlitres of the sample was transferred onto a thin film grid of 100-200 nm. The grid was then plunged into liquid ethane (temperatures below $-170^{\circ} \mathrm{C}$ ) and transferred to a GATAN 626 cryoholder and imaged using a JEOL 2100 transmission electron microscope fitted with a GATAN 4K Ultra scan camera. The cryo TEM analysis was carried out at Unilever, Bedford. 
HPLC

A Dionex P680 HPLC system equipped with a Macherey-Nagel C18 column of $250 \mathrm{~mm}$ length, $4.6 \mathrm{~mm}$ internal diameter and $5 \mathrm{~mm}$ particle size was used to analyze the mixtures of peptide derivatives. The gradient used was a linear exchange between $40 \%$ acetonitrile in water at 4 min to $100 \%$ acetonitrile at $31 \mathrm{~min}$ using a flow rate of $0.7 \mathrm{ml} / \mathrm{min}$ and detection wavelength of 301 $\mathrm{nm}$. Sample preparation: $20 \mu \mathrm{l}$ of sample plus acetonitrile/water $(1.5 \mathrm{ml}, 50: 50 \mathrm{v} / \mathrm{v}$ mixture $)$ containing $0.1 \%$ trifluoroacetic acid.

\section{ACKNOWLEDGMENT}

With thanks to Unilever, Bedford for the use of Cryo-TEM facilities and EPSRC for funding. We acknowledge W. Poon for initial discussions.

Supporting information available: Characterization of enzyme-QD conjugates; additional trajectories; information on QD-AP ratio calculations; calibration curves; Cryo-TEM evidence of fiber formation by QD-AP and QD-AP batch comparison. This material is available free of charge via the Internet at http://pubs.acs.org

\section{REFERENCES}

(1) Godoy, J.; Vives, G.; Tour, J. M. Toward Chemical Propulsion: Synthesis of ROMPPropelled Nanocars. ACS Nano 2011, 5, 85-90.

(2) Gibbs, J. G.; Zhao, Y.-P. Autonomously Motile Catalytic Nanomotors by Bubble Propulsion. Appl. Phys. Lett. 2009, 94, 163104-163104-3.

(3) Sanchez, S.; Solovev, A. A.; Schulze, S.; Schmidt, O. G. Controlled Manipulation of Multiple Cells Using Catalytic Microbots. Chem. Commun. 2010, 47, 698-700.

(4) Wilson, D. A.; de Nijs, B.; van Blaaderen, A.; Nolte, R. J. M.; van Hest, J. C. M. Fuel Concentration Dependent Movement of Supramolecular Catalytic Nanomotors. Nanoscale 2013, 5, 1315.

(5) Laocharoensuk, R.; Burdick, J.; Wang, J. Carbon-Nanotube-Induced Acceleration of Catalytic Nanomotors. ACS Nano 2008, 2, 1069-1075. 
(6) Hong, Y.; Blackman, N. M. K.; Kopp, N. D.; Sen, A.; Velegol, D. Chemotaxis of Nonbiological Colloidal Rods. Phys. Rev. Lett. 2007, 99, 178103.

(7) Paxton, W. F.; Kistler, K. C.; Olmeda, C. C.; Sen, A.; St. Angelo, S. K.; Cao, Y.; Mallouk, T. E.; Lammert, P. E.; Crespi, V. H. Catalytic Nanomotors: Autonomous Movement of Striped Nanorods. J. Am. Chem. Soc. 2004, 126, 13424-13431.

(8) Kline, T. R.; Paxton, W. F.; Mallouk, T. E.; Sen, A. Catalytic Nanomotors: RemoteControlled Autonomous Movement of Striped Metallic Nanorods. Angewandte Chemie 2005, 117, 754-756.

(9) Sanchez, S.; Solovev, A. A.; Mei, Y.; Schmidt, O. G. Dynamics of Biocatalytic Microengines Mediated by Variable Friction Control. J. Am. Chem. Soc. 2010, 132, 13144-13145.

(10) Pantarotto, D.; Browne, W. R.; Feringa, B. L. Autonomous Propulsion of Carbon Nanotubes Powered by a Multienzyme Ensemble. Chem. Commun. 2008, 1533-1535.

(11) Mano, N.; Heller, A. Bioelectrochemical Propulsion. J. Am. Chem. Soc. 2005, 127 , $11574-11575$.

(12) Mansson, A.; Sundberg, M.; Bunk, R.; Balaz, M.; Nicholls, I. A.; Omling, P.; Tegenfeldt, J. O.; Tagerud, S.; Montelius, L. Actin-Based Molecular Motors for Cargo Transportation in Nanotechnology \#8212; Potentials and Challenges. IEEE Transactions on Advanced Packaging 2005, 28, 547-555.

(13) Malcos, J. L.; Hancock, W. O. Engineering Tubulin: Microtubule Functionalization Approaches for Nanoscale Device Applications. Appl. Microbiol. Biotechnol. 2011, 90, 110 .

(14) Wilson, D. A.; Nolte, R. J. M.; van Hest, J. C. M. Autonomous Movement of PlatinumLoaded Stomatocytes. Nat. Chem. 2012, 4, 268-274.

(15) Ismagilov, R. F.; Schwartz, A.; Bowden, N.; Whitesides, G. M. Autonomous Movement and Self-Assembly. Angew. Chem., Int. Ed. 2002, 41, 652-654.

(16) Mitchison, T. J.; Cramer, L. P. Actin-Based Cell Motility and Cell Locomotion. Cell 1996, 84, 371-379.

(17) Pantaloni, D.; Le Clainche, C.; Carlier, M. F. Mechanism of Actin-Based Motility. Science 2001, 292, 1502-1506.

(18) Mogilner, A.; Oster, G. Cell Motility Driven by Actin Polymerization. Biophys. J. 1996, 71, 3030-3045.

(19) Huxley, H. E. Sliding Filaments and Molecular Motile Systems. J. Biol. Chem. 1990, 265, 8347-8350.

(20) Hess, H.; Vogel, V. Molecular Shuttles Based on Motor Proteins: Active Transport in Synthetic Environments. Rev. Mol. Biotechnol. 2001, 82, 67-85.

(21) Williams, R. J.; Smith, A. M.; Collins, R.; Hodson, N.; Das, A. K.; Ulijn, R. V. EnzymeAssisted Self-Assembly under Thermodynamic Control. Nat. Nanotechnol. 2009, 4, 1924.

(22) Olive, A. G. L.; Abdullah, N. H.; Ziemecka, I.; Mendes, E.; Eelkema, R.; van Esch, J. H. Spatial and Directional Control over Self-Assembly Using Catalytic Micropatterned Surfaces. Angew. Chem., Int. Ed. 2014, 53, 4132-4136.

(23) Kuang, Y.; Shi, J.; Li, J.; Yuan, D.; Alberti, K. A.; Xu, Q.; Xu, B. Pericellular Hydrogel/Nanonets Inhibit Cancer Cells. Angew. Chem., Int. Ed. 2014, n/a-n/a. 
(24) Ikezoe, Y.; Washino, G.; Uemura, T.; Kitagawa, S.; Matsui, H. Autonomous Motors of a Metal-organic Framework Powered by Reorganization of Self-Assembled Peptides at Interfaces. Nat. Mater. 2012, 11, 1081-1085.

(25) Reches, M.; Gazit, E. Casting Metal Nanowires within Discrete Self-Assembled Peptide Nanotubes. Science 2003, 300, 625-627.

(26) Zhang, Y.; Gu, H.; Yang, Z.; Xu, B. Supramolecular Hydrogels Respond to Ligand-Receptor Interaction. J. Am. Chem. Soc. 2003, 125, 13680-13681.

(27) Jayawarna, V.; Ali, M.; Jowitt, T. A.; Miller, A. F.; Saiani, A.; Gough, J. E.; Ulijn, R. V. Nanostructured Hydrogels for Three-Dimensional Cell Culture Through Self-Assembly of Fluorenylmethoxycarbonyl-Dipeptides. Adv. Mater. 2006, 18, 611-614.

(28) Mahler, A.; Reches, M.; Rechter, M.; Cohen, S.; Gazit, E. Rigid, Self-Assembled Hydrogel Composed of a Modified Aromatic Dipeptide. Adv. Mater. 2006, 18, 13651370.

(29) Fleming, S.; Debnath, S.; Frederix, P. W. J. M.; Tuttle, T.; Ulijn, R. V. Aromatic Peptide Amphiphiles: Significance of the Fmoc Moiety. Chem. Commun. 2013, 49, 10587-10589.

(30) Bai, S.; Debnath, S.; Gibson, K.; Schlicht, B.; Bayne, L.; Zagnoni, M.; Ulijn, R. V. Biocatalytic Self-Assembly of Nanostructured Peptide Microparticles Using Droplet Microfluidics. Small 2014, 10, 285-293.

(31) Debnath, S.; Roy, S.; Ulijn, R. V. Peptide Nanofibers with Dynamic Instability through Nonequilibrium Biocatalytic Assembly. J. Am. Chem. Soc. 2013, 135, 16789-16792.

(32) Smith, A. M.; Williams, R. J.; Tang, C.; Coppo, P.; Collins, R. F.; Turner, M. L.; Saiani, A.; Ulijn, R. V. Fmoc-Diphenylalanine Self Assembles to a Hydrogel via a Novel Architecture Based on $\pi-\pi$ Interlocked B-Sheets. Adv. Mater. 2008, 20, 37-41.

(33) Chen, L.; Morris, K.; Laybourn, A.; Elias, D.; Hicks, M. R.; Rodger, A.; Serpell, L.; Adams, D. J. Self-Assembly Mechanism for a Naphthalene-Dipeptide Leading to Hydrogelation. Langmuir 2010, 26, 5232-5242.

(34) Ryan, D. M.; Nilsson, B. L. Self-Assembled Amino Acids and Dipeptides as Noncovalent Hydrogels for Tissue Engineering. Polym. Chem. 2012, 3, 18.

(35) Zelzer, M.; Todd, S. J.; Hirst, A. R.; McDonald, T. O.; Ulijn, R. V. Enzyme Responsive Materials: Design Strategies and Future Developments. Biomater. Sci. 2012, 1, 11-39.

(36) Williams, R. J.; Mart, R. J.; Ulijn, R. V. Exploiting Biocatalysis in Peptide Self-Assembly. Biopolymers 2010, 94, 107-117.

(37) Yang, Z; Xu, B. A Simple Visual Assay Based on Small Molecule Hydrogels for Detecting Inhibitors of Enzymes. Chem. Commun. 2004, 2424.

(38) Yang, Z.; Gu, H.; Fu, D.; Gao, P.; Lam, J. K.; Xu, B. Enzymatic Formation of Supramolecular Hydrogels. Adv. Mater. 2004, 16, 1440-1444.

(39) Hughes, M.; Debnath, S.; Knapp, C. W.; Ulijn, R. V. Antimicrobial Properties of Enzymatically Triggered Self-Assembling Aromatic Peptide Amphiphiles. Biomater. Sci. 2013, 1, 1138-1142.

(40) Thornton, K.; Abul-Haija, Y. M.; Hodson, N.; Ulijn, R. V. Mechanistic Insights into Phosphatase Triggered Self-Assembly Including Enhancement of Biocatalytic Conversion Rate. Soft Matter 2013, 9, 9430-9439.

(41) Yang, Z.; Liang, G.; Wang, L.; Xu, B. Using a Kinase/Phosphatase Switch to Regulate a Supramolecular Hydrogel and Forming the Supramolecular Hydrogel in vivo. J. Am. Chem. Soc. 2006, 128, 3038-3043. 
(42) Abul-Haija, Y. M.; Roy, S.; Frederix, P. W. J. M.; Javid, N.; Jayawarna, V.; Ulijn, R. V. Biocatalytically Triggered Co-Assembly of Two-Component Core/Shell Nanofibers. Small 2013, n/a-n/a.

(43) Li, J.; Gao, Y.; Kuang, Y.; Shi, J.; Du, X.; Zhou, J.; Wang, H.; Yang, Z.; Xu, B. Dephosphorylation of D-Peptide Derivatives to Form Biofunctional, Supramolecular Nanofibers/Hydrogels and Their Potential Applications for Intracellular Imaging and Intratumoral Chemotherapy. J. Am. Chem. Soc. 2013, 135, 9907-9914.

(44) Gao, Y.; Shi, J.; Yuan, D.; Xu, B. Imaging Enzyme-Triggered Self-Assembly of Small Molecules inside Live Cells. Nat. Commun. 2012, 3, 1033.

(45) Sadownik, J. W.; Leckie, J.; Ulijn, R. V. Micelle to Fibre Biocatalytic Supramolecular Transformation of an Aromatic Peptide Amphiphile. Chem. Commun. 2011, 47, 728-730.

(46) Gonçalves, M. S. T. Fluorescent Labeling of Biomolecules with Organic Probes. Chem. Rev. 2009, 109, 190-212.

(47) Lo, W.; Rodgers, W.; Hughes, T. Making Genes Green: Creating Green Fluorescent Protein (GFP) Fusions with Blunt-End PCR Products. BioTechniques 1998, 25, 94-96, 98.

(48) Nan, X.; Sims, P. A.; Xie, X. S. Organelle Tracking in a Living Cell with Microsecond Time Resolution and Nanometer Spatial Precision. Chemphyschem 2008, 9, 707-712.

(49) Biebricher, A.; Wende, W.; Escudé, C.; Pingoud, A.; Desbiolles, P. Tracking of Single Quantum Dot Labeled EcoRV Sliding along DNA Manipulated by Double Optical Tweezers. Biophys. J. 2009, 96, L50-52.

(50) Zhu, H.; Srivastava, R.; Brown, J. Q.; McShane, M. J. Combined Physical and Chemical Immobilization of Glucose Oxidase in Alginate Microspheres Improves Stability of Encapsulation and Activity. Bioconjug. Chem. 2005, 16, 1451-1458.

(51) Sowa, Y.; Steel, B. C.; Berry, R. M. A Simple Backscattering Microscope for Fast Tracking of Biological Molecules. Rev. Sci. Instrum. 2010, 81, 113704.

(52) Jamieson, T.; Bakhshi, R.; Petrova, D.; Pocock, R.; Imani, M.; Seifalian, A. M. Biological Applications of Quantum Dots. Biomaterials 2007, 28, 4717-4732.

(53) Sperling, R. A.; Rivera Gil, P.; Zhang, F.; Zanella, M.; Parak, W. J. Biological Applications of Gold Nanoparticles. Chem. Soc. Rev. 2008, 37, 1896.

(54) Green, M.; O'Brien, P. Recent Advances in the Preparation of Semiconductors as Isolated Nanometric Particles: New Routes to Quantum Dots. Chem. Commun. 1999, 2235-2241.

(55) Smith, A. M.; Duan, H.; Mohs, A. M.; Nie, S. Bioconjugated Quantum Dots for in vivo Molecular and Cellular Imaging. Adv. Drug Deliv. Rev. 2008, 60, 1226-1240.

(56) Jaiswal, J. K.; Simon, S. M. Potentials and Pitfalls of Fluorescent Quantum Dots for Biological Imaging. Trends Cell Biol. 2004, 14, 497-504.

(57) McHale, K.; Berglund, A. J.; Mabuchi, H. Quantum Dot Photon Statistics Measured by Three-Dimensional Particle Tracking. Nano Lett. 2007, 7, 3535-3539.

(58) Holtz, K. M.; Stec, B.; Kantrowitz, E. R. A Model of the Transition State in the Alkaline Phosphatase Reaction. J. Biol. Chem. 1999, 274, 8351-8354.

(59) Sengupta, S.; Dey, K. K.; Muddana, H. S.; Tabouillot, T.; Ibele, M. E.; Butler, P. J.; Sen, A. Enzyme Molecules as Nanomotors. J. Am. Chem. Soc. 2013, 135, 1406-1414.

(60) Wang, W.; Chau, Y. Self-Assembled Peptide Nanorods as Building Blocks of Fractal Patterns. Soft Matter 2009, 5, 4893-4898.

(61) Albertazzi, L.; van der Zwaag, D.; Leenders, C. M. A.; Fitzner, R.; van der Hofstad, R. W.; Meijer, E. W. Probing Exchange Pathways in One-Dimensional Aggregates with Super-Resolution Microscopy. Science 2014, 344, 491-495. 
(62) Alegre-Cebollada, J.; Perez-Jimenez, R.; Kosuri, P.; Fernandez, J. M. Single-Molecule Force Spectroscopy Approach to Enzyme Catalysis. J. Biol. Chem. 2010, 285, 1896118966.

(63) Mori, T.; Vale, R. D.; Tomishige, M. How Kinesin Waits between Steps. Nature 2007, 450.

(64) Invitrogen. Qdot ITK Carboxyl Quantum Dots, 2007.

(65) Crocker, J. C.; Grier, D. G. Methods of Digital Video Microscopy for Colloidal Studies. J. Colloid Interface Sci. 1996, 179, 298-310. 